\title{
HOMOLOGY AND IMAGES OF SEMIANALYTIC SETS
}

\author{
BY ROBERT M. HARDT ${ }^{1}$
}

Communicated by William Browder, January 3, 1974

\begin{abstract}
The homology of semianalytic sets may be treated using chains which are themselves locally-finite integral combinations of disjoint, oriented semianalytic submanifolds. The analytic image of a relatively compact semianalytic set, though not necessarily semianalytic, admits a finite stratification into connected analytic submanifolds of various dimensions.
\end{abstract}

A subset $A$ of a (real) analytic manifold $M$ is called analytic (respectively, semianalytic) if $M$ can be covered by open sets $U$ for which there is a real-valued function $f$ (respectively, a finite family $\mathscr{F}$ of real-valued functions) analytic in $U$ so that $U \cap A$ equals $f^{-1}\{0\}$ (respectively, $U \cap A$ is a union of connected components of $f^{-1}\{0\} \sim g^{-1}\{0\}$ for some $f, g \in \mathscr{F}$ ). A stratum in $M$ is a connected (properly embedded) differentiable submanifold of $M$. A stratification $\mathscr{S}$ of a subset $A$ of $M$ is a locally finite partition of $A$ into strata $\mathscr{S}$ so that $(A \cap \operatorname{Clos} S) \sim S$ is a union of strata in $\mathscr{S}$ having dimension less than the dimension of $S$. It is well known $[9, \S 13],[7,2.8]$ that every semianalytic set admits a stratification into semianalytic strata.

A $j$-dimensional analytic chain $T$ in $M$ is a sum of integral multiples of oriented $j$-dimensional semianalytic strata belonging to some fixed stratification of $M$. Since the restriction to these strata of $j$-dimensional Hausdorff measure is locally-finite by $[2,3.4 .8(13)]$, the analytic chain $T$ is (by oriented integration, counting multiplicities, of differential $j$ forms of compact support in $M$ ) a $j$-dimensional current in $M$. The set spt $T$, being the union of the closures of the strata occurring with nonzero multiplicity, is semianalytic. For $j \geqq 1$, the $(j-1)$-dimensional current $\partial T$, defined by $\partial T(\psi)=T(d \psi)$ for $\psi \in \mathscr{D}^{j-1}(M)$, is, by [2, 4.2.28], also an analytic chain in $M$.

Suppose $M \supset A \supset B$. Using the group of real analytic cycles $\mathscr{Z}_{j}(A, B)=$ $\{T: T$ is a $j$-dimensional analytic chain of compact support, spt $T \subset A$,

AMS (MOS) subject classifications (1970). Primary 32C05, 32C25; Secondary 55B35, 55B45, 28A75.

Key words and phrases. Semianalytic set, stratum, stratification, analytic chain, current, real analytic homology group, semianalytic shadow.

1 Research partially supported by NSF Grant GP29321. 
and spt $\partial T \supset B$ or $j=0\}$, the subgroup of real analytic boundaries, $\mathscr{B}_{j}(A, B)=\left\{R+\partial S: R \in \mathscr{Z}_{j}(B, B)\right.$ and $\left.S \in \mathscr{Z}_{j+1}(A, A)\right\}$, and the real analytic homology group, $\boldsymbol{H}_{j}(A, B)=\mathscr{Z}_{j}(A, B) / \mathscr{B}_{j}(A, B)$, we obtain in $[6,4.6-4.7]$ the following

THEOREM 1. If $A \supset B$ are semianalytic sets, then there exists an arbitrarily small open neighborhood $W$ of $B$ such that $\boldsymbol{H}_{j}(A \cap W, B) \simeq 0$ for all $j$.

COROLlARY 1. There exist arbitrarily small open neighborhoods $U$ of $A$ in $M$ and $V$ of $B$ in $U$ so that the inclusion of $\mathscr{Z}_{j}(A, B)$ into $\mathscr{Z}_{j}(U, V)$ induces an isomorphism, $\boldsymbol{H}_{j}(A, B) \simeq \boldsymbol{H}_{j}(U, V)$, for all $j$.

This allows us to define in $[6, \S 5]$, by approximation, the homomorphism $\boldsymbol{H}_{j}(f): \boldsymbol{H}_{j}(C, D) \rightarrow \boldsymbol{H}_{j}(A, B)$ for any continuous map $f:(C, D) \rightarrow$ $(A, B)$ where $C \supset D$ are semianalytic subsets of an analytic manifold; the axioms of Eilenberg-Steenrod follow as in [2, 4.4.1]. Also in [6, §6] a homology intersection product, for oriented $M$,

$$
\bigcap: H_{i}(A, B) \times H_{j}(A, B) \rightarrow H_{i+j-\operatorname{dim} M}(A, B)
$$

where $i+j \geqq \operatorname{dim} M$, results by use of the intersection theory for real analytic chains of $[4, \S 5]$. The proofs of $[6, \S \S 2-6]$ all carry over for an analogous treatment of the homology of semialgebraic sets by real algebraic chains or for the homology with $Z / \nu Z$ coefficients, where $\nu \in\{2,3 \cdots\}$, of semianalytic sets by real analytic chains modulo $v$ [5].

A. Borel and A. Haefliger, employing the Borel-Moore homology for locally-compact spaces proved the orientability modulo 2 of real analytic sets and established a formula equating the modulo 2 cycle of the real part of the intersection of two holomorphic varieties with the intersection of the modulo 2 cycles of the real parts of the varieties. These facts are reproven in $[6, \S \S 6,8]$ using analytic chains and Federer's theory of slicing $[2,4.3],[4, \S 4]$. The proof of Theorem 1 involves, for bounded semianalytic subsets of $\boldsymbol{R}^{n}$, a certain stratification and system of neighborhoods built up from finitely many local normal decompositions of Łojasiewicz [9, §13]. We do not make use of the triangulability of semianalytic sets which is established in [3] and [8]. Other interesting aspects of semianalytic sets are treated in $[1],[2,3.4 .5-3.4 .11],[9],[10],[11, \S 4]$ and [12], [9] being the most informative.

Even though semianalytic sets are closed under finite union, intersection, complement, cartesian product, and inverse image under analytic maps, the analytic image of even a compact analytic manifold may fail to be semianalytic [9, p. 135]. A subset $C$ of $M$ is called a semianalytic shadow if $M$ can be covered by open sets $U$ for which there is an analytic manifold $P$, an analytic mapping $p: P \rightarrow M$, and a finite family $\mathscr{A}$ of 
relatively compact semianalytic subsets of $P$ so that $U \cap C$ is a union of connected components of $p(A) \sim p(B)$ for some $A, B \in \mathscr{A}$.

THEOREM 2. For any locally finite family $\mathscr{C}$ of semianalytic shadows in $M$, there is a stratification $\mathscr{S}$ of $M$ into semianalytic shadows so that each member of $\mathscr{C}$ is a union of strata in $\mathscr{S}$.

THEOREM 3. If $f: M \rightarrow N$ is a proper analytic mapping, then $\mathscr{S}$ may be chosen so that $\{f(S): S \in \mathscr{S}\}$ extends to a stratification of $N$ and $f \mid S$ is one-one for all $S \in \mathscr{S}$ with $\operatorname{dim} f(S)=\operatorname{dim} S$.

The proofs of these statements in [7] involve certain semianalytic stratifications, the rank theorem, a cartesian product construction of $[4,2.8]$, and induction on the Hausdorff dimension of $\bigcup \mathscr{C}$. The stratification $\mathscr{S}$ may also be refined to satisfy Whitney condition (B) [11, §3]. Statements similar to Theorem 2 and Corollary 2 are given without formal proof in [12, III B-C].

We have recently learned of the interesting work of $H$. Hironaka [13] and [14] on semianalytic shadows (which he calls subanalytic sets). Using his theory of resolution of singularities and blowing up techniques, he has [13] established Theorem 2. Because of differences in proofs as well as the discussions of $[7, \S 2, \S 5]$, our article [7] may be of independent interest.

\section{REFERENCES}

1. D. Burghelea and A. Verona, Local homological properties of analytic sets, Manuscripta Math. 7 (1972), 55-66.

2. H. Federer, Geometric measure theory, Die Grundlehren der math. Wissenschaften, Band 153, Springer-Verlag, Berlin and New York, 1969. MR 41 \#1976.

3. B. Giesecke, Simpliziale Zerlegung abzählbarer analytischer Räume, Math. Z. 83 (1964), 177-213. MR 28 \#2563.

4. R. Hardt, Slicing and intersection theory for chains associated with real analytic varieties, Acta Math. 129 (1972), 75-136.

5. — Slicing and intersection theory for chains modulo $v$ associated with real analytic varieties, Trans. Amer. Math. Soc. 183 (1973), 327-340.

6. - Homology theory for real analytic and semianalytic sets, Ann. Scuola Norm. Sup. Pisa (to appear).

7. - Stratification of real analytic mappings and images, (preprint).

8. S. Łojasiewicz, Triangulation of semi-analytic sets, Ann. Scuola Norm. Sup. Pisa (3) 18 (1964), 449-474. MR 30 \#3478.

9. — Ensembles semianalytiques, Cours Faculté des Sciences d'Orsay, Inst. Haute Etudes Sci., Bures-sur-Yvette, 1965.

10. — Une propiété topologique des sous-ensembles analytique reéls, Colloques internationaux du Centre National de la Recherche Scientifique, No. 117, Les Equations aux Dérivées Partielles, Paris, 1972, pp. 87-89.

11. J. Mather, Stratifications and mappings, Harvard University notes, Cambridge, Mass., 1971. 
12. R. Thom, Ensembles et morphismes stratifiés, Bull. Amer. Math. Soc. 75 (1969), 240-284. MR 39 \#970.

13. H. Hironaka, Subanalytic sets, Number Theory, Algebraic Geometry, and Commutative Algebra (Dedicated to Akizuki), Kinokunia, Tokyo, Japan, 1973, pp. 453-493.

14. - Subanalytic sets, Lecture notes of Istituto matematico, Leonida Tonelli, Pisa, 1973.

School of Mathematics, University of Minnesota, Minneapolis, Minnesota 55455 